\title{
ON EXTENSIONS OF AN INEQUALITY AMONG MEANS ${ }^{1}$
}

\author{
F. CHAN, ${ }^{2}$ D. GOLDBERG AND S. GONEK
}

Dedicated to the family of Frank Chan, in the hope that this paper will somehow ease the loss due to his untimely death.

ABstract. An inequality of Fan relates the arithmetic and geometric means of $x$ and $1-x$. An extension to generalized means is conjectured. This conjecture is proven for several special cases. In addition, some counterexamples are given.

1. Introduction. The following inequality is due to Ky Fan [1]

$$
\left[\frac{\prod_{i=1}^{n} x_{i}}{\prod_{i=1}^{n}\left(1-x_{i}\right)}\right]^{1 / n}<\frac{\sum_{i=1}^{n} x_{i}}{\sum_{i=1}^{n}\left(1-x_{i}\right)}, \quad 0 \leqq x_{i} \leqq \frac{1}{2},
$$

unless $x_{1}=x_{2}=\cdots=x_{n}$. Recently there have been generalizations of (1) ([3], [4], and [5]). Presented here are the results of investigations into a still different generalization, originally suggested by D. Segalman.

With the notation

$$
\|x\|_{D}=\left[\frac{1}{n} \sum_{i=1}^{n}\left|x_{i}\right|^{p}\right]^{1 / p} \text { and }\|x\|_{0}=\lim _{p \rightarrow 0}\|x\|_{p}=\left[\prod_{i=1}^{n}\left|x_{i}\right|\right]^{1 / n},
$$

(1) becomes

$$
\|x\|_{0} /\|1-x\|_{0}<\|x\|_{1} /\|1-x\|_{1},
$$

with the appropriate conditions on $x$. Since the theorem of the arithmetic and geometric means, $\|x\|_{0}<\|x\|_{1}$ generalizes to $\|x\|_{p}<\|x\|_{q}, p<q$, one might conjecture that one can similarly extend (2) to

$$
\|x\|_{p} /\|1-x\|_{p}<\|x\|_{Q} /\|1-x\|_{Q}, \quad p<q .
$$

2. The two dimensional case. When $n=2$, equation (3) holds.

Received by the editors February 19, 1973.

AMS (MOS) subject classifications (1970). Primary 26A86.

Key words and phrases. Inequality among means, generalized means, arithmetic means, geometric means.

'Supported by the National Science Foundation.

'Deceased. 
THEOREM 1. If $0 \leqq x, 0 \leqq y, x+y<1, x<y$ and $p<q$, then

$$
\left[\frac{x^{p}+y^{p}}{(1-x)^{p}+(1-y)^{p}}\right]^{1 / p}<\left[\frac{x^{q}+y^{q}}{(1-x)^{q}+(1-y)^{q}}\right]^{1 / q} \text {. }
$$

The cases where $p$ and $q$ are infinite or zero are not excluded.

Proof. First assume that $0<p<q<\infty$ or $-\infty<p<q<0$, and that $0<x$ and write (4) as

$$
\frac{\left[1+(y / x)^{p}\right]^{q}}{\left[1+(y / x)^{q}\right]^{p}}<\frac{\left[1+((1-y) /(1-x))^{p}\right]^{q}}{\left[1+((1-y) /(1-x))^{q}\right]^{p}} .
$$

Introducing the new variables $r=y / x, s=(1-y) /(1-x)$ leads to the conditions

$$
0 \leqq r, \quad 0 \leqq s, \quad r>1, \quad s<1, \quad r s>1 .
$$

The last inequality follows from the equivalence of $\left(y-y^{2}\right) /\left(x-x^{2}\right)>1$ with $(x-y)(1-(x+y))<0$. The function defined by $f(z)=\left(1+z^{p}\right)^{q} /\left(1+z^{q}\right)^{p}$ has these properties:

(7) $f(z)=f(1 / z), \quad f^{\prime}(z)=\left[p q z^{-1}\left(1+z^{p}\right)^{a-1}\left(1+z^{Q}\right)^{-p-1}\right]\left[z^{D}-z^{Q}\right]$.

Since (5) is equivalent to $f(r)<f(s)$, by (7) we need only show that $f(1 / r)<f(s)$. But by (6), $0<1 / r<s<1$; so since (7) shows that $f^{\prime}(z)>0$, $0<z<1$, (5) is established. By taking limits, one has $\|z\|_{q} \leqq\|z\|_{0}$ and $\|z\|_{0} \leqq\|z\|_{p}$, where $z=(x, y)$. Choosing an $r$ with $q<r<0$ gives $\|z\|_{q}<$ $\|z\|_{r} \leqq\|z\|_{0} \leqq\|z\|_{p}$, proving (5) for $q<0<p$. All the other excluded cases of $p$ and $q$ are proven in precisely the same manner. When $x=0,(5)$ is equivalent to $f(z)>1$ for $z>0$.

3. Some counterexamples. The conjecture (3) is not true for arbitrary $n$. In some cases, one has

$$
\left[\frac{\sum_{i=1}^{n} x_{i}^{p}}{\sum_{i=1}^{n}\left(1-x_{i}\right)^{p}}\right]^{1 / p}>\left[\frac{\sum_{i=1}^{n} x_{i}^{q}}{\sum_{i=1}^{n}\left(1-x_{i}\right)^{q}}\right]^{1 / q}, \quad p<q, 0 \leqq x_{i} \leqq \frac{1}{2} .
$$

Let $x_{1}=x_{2}=\cdots=x_{n}=\frac{1}{2}$, and $x_{n+1}=0$. Then (8) becomes

$$
\left[1+2^{p} / n\right]^{1 / p}<\left[1+2^{q} / n\right]^{1 / a} .
$$

If one defines $f_{n}(p)=\left[1+2^{p} / n\right]^{1 / p}$, then (9) will hold for $\bigwedge_{n}<p<q$ if $f_{n}^{\prime}(p)>0$ for $p>\wedge_{n}$. Computation shows that $f_{n}^{\prime}(p)>0$ if and only if

$$
g_{n}(p)=\left(p 2^{p} / n\right) \log 2-\left(1+2^{p} / n\right) \log \left(1+2^{p} / n\right)>0 .
$$


Now make the variable change

$$
x=1+2^{p} / n, \quad p=\log (n(x-1)) / \log 2
$$

and let $g_{n}(p)=h_{n}(x)=(x-1) \log (n(x-1))-x \log x$. Since

$$
h_{n}^{\prime}(x)=\log (n(x-1))-\log x \text { and } h_{n}^{\prime \prime}(x)=1 / x(x-1),
$$

for $x>n /(n-1)>1, h_{n}^{\prime}(x)>0$ and $h_{n}^{\prime \prime}(x)>0$. The relation $h_{n}(n /(n-1))=$ $-\log (n /(n-1))<0$ shows that for $\lambda_{n}>n /(n-1)$ with $h_{n}\left(\lambda_{n}\right)=0$, we can choose

$$
\bigwedge_{n}=\log \left(n\left(\lambda_{n}-1\right)\right) / \log 2
$$

It is easily seen that $\bigwedge_{2}<3, \bigwedge_{4}=2$, and in fact $\bigwedge_{n}>\bigwedge_{n+1}, n=4,5$, $6, \cdots$. This follows from

$$
\frac{d}{d n} g_{n}(p)=\frac{2^{p}}{n^{2}}\left[1+\log \left(1+\frac{2^{p}}{n}\right)-p \log 2\right]
$$

which forces $(d / d n) g_{n}(p)>0$ if and only if $e>2^{p}(1-e / n)$, together with $\bigwedge_{4}=2$ and $\left.(d / d n) g_{n}(2)\right|_{n=4}>0$.

REMARKS. If (9) is expanded by the binomial theorem, one obtains

$$
1+2^{p} / p n+O\left(1 / n^{2}\right)<1+2^{q} / q n+O\left(1 / n^{2}\right) .
$$

Thus for $n$ sufficiently large, (8) holds provided $2^{p} / p<2^{q} / q$. Examination of the graph of $2^{p} / p$ gives a useful picture, and since it has a maximum at $(\log 2)^{-1}$, one can conclude that $(\log 2)^{-1}<\bigwedge_{n}$.

In the special case of (3) where $n=3$, the expansion of the equivalent inequality

$$
\begin{aligned}
& {\left[\frac{(1-u)^{p}+(1-v)^{p}+(1-w)^{p}}{(1+u)^{p}+(1+v)^{p}+(1+w)^{p}}\right]^{1 / p}} \\
& <\left[\frac{(1-u)^{Q}+(1-v)^{a}+(1-w)^{Q}}{(1+u)^{Q}+(1+v)^{Q}+(1+w)^{Q}}\right]^{1 / \alpha}
\end{aligned}
$$

with $u=v=0$ through cubic powers in $w$ gives $p+q \leqq 9$, establishing (8) for $p+q>9$, and $u$ sufficiently small.

4. Two $n$-dimensional theorems. There are two other cases in which (3) holds.

THEOREM 2. If $0 \leqq x_{i} \leqq \frac{1}{2}$ and if $p>0$, then

$$
\left[\frac{\sum_{i=1}^{n} x_{i}^{-D}}{\sum_{i=1}^{n}\left(1-x_{i}\right)^{-D}}\right]^{-1 / p}<\left[\frac{\sum_{i=1}^{n} x_{i}^{p}}{\sum_{i=1}^{n}\left(1-x_{i}\right)^{p}}\right]^{1 / p}
$$

unless $x_{1}=x_{2}=\cdots=x_{n}$. 
Proof. First write (12) as

$$
\sum_{i=1}^{n} \frac{1}{\left(1-x_{i}\right)^{p}} \sum_{i=1}^{n}\left(1-x_{i}\right)^{p}<\sum_{i=1}^{n} \frac{1}{x_{i}^{p}} \sum_{i=1}^{n} x_{i}^{p} .
$$

When $n=2$, (13) is a special case of Theorem 1. To prove (13) for arbitrary $n$, consider the $n(n-1) / 2$ inequalities

$$
\begin{aligned}
\left(\frac{1}{\left(1-x_{i}\right)^{p}}+\frac{1}{\left(1-x_{j}\right)^{p}}\right) & \left(\left(1-x_{i}\right)^{p}+\left(1-x_{j}\right)^{p}\right) \\
& <\left(\frac{1}{x_{i}^{p}}+\frac{1}{x_{j}^{p}}\right)\left(x_{i}^{p}+x_{j}^{p}\right), \quad 1 \leqq i<j \leqq n .
\end{aligned}
$$

If these are summed together, with the exception of constant terms every term in (13) will appear exactly once in the sum. Since the same constant term appears in each side of (13) and (14), this proves the theorem.

THEOREM 3. If $0 \leqq p \leqq 1$ and $1 \leqq q \leqq 2$ and $p \neq q$, then for $0 \leqq x_{i} \leqq \frac{1}{2}$,

$$
\left[\frac{\sum_{i=1}^{n} x_{i}^{p}}{\sum_{i=1}^{n}\left(1-x_{i}\right)^{p}}\right]^{1 / p}<\left[\frac{\sum_{i=1}^{n} x_{i}^{q}}{\sum_{i=1}^{n}\left(1-x_{i}\right)^{q}}\right]^{1 / q},
$$

unless $x_{1}=x_{2}=\cdots=x_{n}$.

Proof. Suppose $p=1$ and $1<q<2$, and suppose $x_{i}=a \neq x_{j}=b$, for some $i \neq j$. If we can prove the following (here. $i=n-1, j=n$ )

$$
\begin{aligned}
& \frac{\sum_{i=1}^{n-2} x_{i}^{a}+2((a+b) / 2)^{a}}{\sum_{i=1}^{n-2}\left(1-x_{i}\right)^{a}+2(1-(a+b) / 2)^{a}} \sum_{i=1}^{n-2} x_{i}^{a}+a^{a}+b^{a} \\
&<\frac{\sum_{i=1}^{n-2}\left(1-x_{i}\right)^{a}+(1-a)^{a}+(1-b)^{a}}{}
\end{aligned}
$$

then the proof will be complete. This method is explained in detail in [2, $\S 2.6]$ and [3].

Next let $\sum$ stand for $\sum_{i=1}^{n-2}$ and break (16) into two pieces

$$
\text { 17) } \begin{aligned}
\sum x_{i}^{q}\left[(1-a)^{a}+(1-b)^{a}\right. & \left.-2(1-(a+b) / 2)^{a}\right] \\
& <\sum\left(1-x_{i}\right)^{a}\left[a^{a}+b^{q}-2((a+b) / 2)^{a}\right],
\end{aligned}
$$

Note that (18) is a special case of Theorem 1. Since $\sum x_{i}^{q}<\sum\left(1-x_{i}\right)^{q}$ and $(1-a)^{q}+(1-b)^{q} \geqq 2(((1-a)+(1-b)) / 2)^{q}$ (Jensen's inequality), (17) will follow from

$$
\begin{aligned}
\frac{1}{2}(1-a)^{a}+\frac{1}{2}(1-b)^{a} & -\left[\frac{1}{2}(1-a)+\frac{1}{2}(1-b)\right]^{a} \\
& <\frac{1}{2} a^{a}+\frac{1}{2} b^{a}-\left[\frac{1}{2} a+\frac{1}{2} b\right]^{a} .
\end{aligned}
$$


But this is Levinson's generalization [4], with $f(x)=x^{q}$ and $p_{1}=p_{2}=\frac{1}{2}$. Levinson's result is stated in the appendix. When $q=1,0<p<1,(17)$ and (18) both reverse. These two inequalities establish the theorem.

5. More general conditions. Some of the preceding theorems hold true under more general conditions than $0 \leqq x_{i} \leqq \frac{1}{2}$. Theorem 2 is true if $x_{i}+x_{j} \leqq 1$ for all $1 \leqq i, j \leqq n$, and Theorem 3 holds if $x_{i}+x_{n+1-i} \leqq 1$, $1 \leqq i \leqq n$, for some ordering of the $x_{i}$ 's. The proofs are exactly the same. When $p=1$ and $q=2(15)$ becomes

$$
\left[\frac{\sum_{i=1}^{n} x_{i}}{n-\sum_{i=1}^{n} x_{i}}\right]^{2}<\frac{\sum_{i=1}^{n} x_{i}^{2}}{\sum_{i=1}^{n}\left(1-x_{i}\right)^{2}}
$$

This is equivalent to $\left(n-2 \sum_{i=1}^{n} x_{i}\right)\left(n \sum_{i=1}^{n} x_{i}^{2}-\left(\sum_{i=1}^{n} x_{i}\right)^{2}\right)>0$. If $x_{1}=$ $x_{2}=\cdots=x_{n}$ does not hold, the identity

$$
\sum_{i=1}^{n}\left(x_{i}-\bar{x}\right)^{2}=\sum_{i=1}^{n} x_{i}^{2}-\frac{\left(\sum_{i=1}^{n} x_{i}\right)^{2}}{n}
$$

where $\bar{x}=\left(\sum_{i=1}^{n} x_{i}\right) / n$ shows that $(20)$ is true if and only if $\sum_{i=1}^{n} x_{i}<n / 2$.

6. Conclusions. Although the anomalous behavior of the conjecture $\|x\|_{\mathcal{D}} /\|1-x\|_{\mathcal{D}}<\|x\|_{a} /\|1-x\|_{a}, p<q$, suggests that it may not be a fruitful object for study, it raises some interesting questions. Most classical inequalities follow inductively from the two dimensional theorem (see [2, §2.14]). This conjecture does not. Perhaps the "right" theorem contains ordering conditions which are trivially satisfied in two dimensions. The counterexamples of $\S 3$ show that our theorems are close to the best possible, except for the case of negative $p$ and $q$.

7. Appendix. Below is the theorem from [4].

THEOREM (LEVINSON). Let $f$ be a three times differentiable function, with $f^{\prime \prime \prime}(x)>0$ for $x \in(0,2 a), 0 \leqq x_{i} \leqq a$, and $0<p_{i}, i=1,2, \cdots, n$. Then, unless $x_{1}=x_{2}=\cdots=x_{n}$,

$\frac{\sum_{i=1}^{n} p_{i} f\left(x_{i}\right)}{\sum_{i=1}^{n} p_{i}}-f\left[\frac{\sum_{i=1}^{n} p_{i} x_{i}}{\sum_{i=1}^{n} p_{i}}\right]<\frac{\sum_{i=1}^{n} p_{i} f\left(2 a-x_{i}\right)}{\sum_{i=1}^{n} p_{i}}-f\left[\frac{\sum_{i=1}^{n} p_{i}\left(2 a-x_{i}\right)}{\sum_{i=1}^{n} p_{i}}\right]$.

The inequality reverses if $f^{\prime \prime \prime}(x)<0$. This is slightly more general than Levinson's paper, but the proof is the same.

ACKNOWLeDgement. The authors would like to thank Professor Richard Askey for his guidance during the course of the investigations, and for his valuable assistance in preparing this paper. 


\section{REFERENCES}

1. E. F. Beckenbach and R. Bellman, Inequalities, 2nd ed., Ergebnisse der Math. und ihrer Grenzgebiete, Band 30, Springer-Verlag, New York, 1965, p. 5. MR 33 \#236.

2. G. H. Hardy, J. E. Littlewood and G. Pólya, Inequalities, 2nd ed., Cambridge Univ. Press, New York, 1952. MR 13, 727.

3. S. Lawrence and D. Segalman, A generalization of two inequalities involving means, Proc. Amer. Math. Soc. 35 (1972), 96-100.

4. N. Levinson, Generalization of an inequality of Ky Fan, J. Math. Anal. Appl. 8 (1964), 133-134. MR 28 \#171.

5. T. Popoviciu, Sur une inégalité de N. Levinson, Mathematica 6 (1964), 301-306.

Department of Mathematics, University of Wisconsin, Madison, Wisconsin 53706

Current address (S. Gonek): Department of Mathematics, University of Michigan, Ann Arbor, Michigan 48104 